\title{
Digitalisierung im gesundheitlichen Verbraucherschutz - eine neue Dimension?
}

\author{
Helmut Tschiersky ${ }^{1}$
}

Published online: 3 November 2018

(C) Bundesamt für Verbraucherschutz und Lebensmittelsicherheit (BVL) 2018

Digitalisierung ist in aller Munde. Täglich steht dazu etwas in den Zeitungen, die Bundesregierung will die Digitalisierung vorantreiben, Ministerien stellen Digitalisierungsreferenten ein, Unternehmensberatungen veranstalten dazu Fortbildungen und die Wissenschaft publiziert Forschungsergebnisse, die vor wenigen Jahren aufgrund der Datenmengen noch gar nicht generierbar gewesen wären. Ein einheitliches Digitalisierungskonzept fehlt jedoch.

Digitalisierung im ursprünglichen Sinne ist die Umwandlung von analogen Werten in digitale Formate, die sich dann informationstechnisch verarbeiten lassen. So gesehen ist Digitalisierung schon lange im gesundheitlichen Verbraucherschutz etabliert. Seit Jahrzehnten produzieren Analysengeräte in den amtlichen Untersuchungslaboren digitalisierte Messwerte, die über Auswertesysteme weiter aufbereitet werden. Ein strukturiertes Datenmeldeportal leitet die Ergebnisse an das Bundesamt für Verbraucherschutz und Lebensmittelsicherheit (BVL) weiter, wo sie wiederum für die wissenschaftliche Bewertung durch das Bundesinstitut für Risikobewertung (BfR) verfügbar gemacht werden. So führen koordinierte Überwachungsprogramme $\mathrm{zu}$ wichtigen Erkenntnissen im gesundheitlichen Verbraucherschutz. Ein anderes Beispiel betrifft das Fachinformationssystem Verbraucherschutz/ Lebensmittelsicherheit (FIS-VL), ein Portal, in dem verschiedene Behörden Dokumente einstellen und Informationen austauschen können. Ein unverzichtbares Instrument zur Bewältigung von Lebensmittelereignissen und -krisen, weil es wichtige Zeit spart, allen Beteiligten gleichzeitig zugänglich ist und ein Krisenmanagement quasi ,in Echtzeit" ermöglicht.

Helmut Tschiersky

helmut.tschiersky@bvl.bund.de

1 Bundesamt für Verbraucherschutz und Lebensmittelsicherheit (BVL), Berlin, Germany

\section{Vernetzung 4.0 im gesundheitlichen Verbraucherschutz}

Aber die Digitalisierung ist weit mehr als die Umwandlung von Daten. Dr. Georg Schreiber hat in diesem Journal schon seine Vision zur Lebensmittelkontrolle und Verbraucherinformation im digitalen Zeitalter beschrieben (Schreiber 2013). Seither haben sich die gesellschaftlichen Rahmenbedingungen - weg von einer Präsenzkultur hin zu einer digitalen Kommunikation - weiterentwickelt. Mehr denn je möchten die Menschen ihre Arbeits- und Freizeiten frei bestimmen. Starre Öffnungszeiten im Lebensmittelhandel stehen dem entgegen und fördern den Onlinekauf, spontan und von überall mit dem eigenen Smartphone. Gleichzeitig erwarten Verbraucher Informationen und Transparenz. Nicht nur zum Preis sondern auch zu Herkunft und Bewertung.

Konsequenterweise findet die Kommunikation zu Verbraucherthemen heute auch in den sozialen Medien statt, dem digitalen Wohnzimmer des Verbrauchers. Die Netzwerke bieten Behörden die Chance, direkt mit Nutzern zu Themen der Lebensmittelsicherheit ins Gespräch zu kommen. Im Falle einer Lebensmittelkrise können Behörden die Verbraucher mithilfe sozialer Medien schnell informieren. Jedoch erwarten die Nutzer Reaktionen in Echtzeit. Werden diese Erwartungen nicht erfüllt, kann es schnell zu einem sogenannten „Shitstorm“ kommen.

\section{Auswirkungen für die behördliche Kommunikation}

Daher sollte jede Behörde im gesundheitlichen Verbraucherschutz ihre Kommunikationsstrategie weiterentwickeln, um den neuen Kommunikationsbedürfnissen der Verbraucher gerecht zu werden. Die Behörden der Bundesländer und das BVL haben sich auf diese Entwicklungen eingestellt und sorgen dafür, dass virtuelle Marktplätze genauso sicher sind wie der Laden um die 
Ecke. Seit 2013 betreiben die Bundesländer hierfür beim BVL die Internetkontrollstelle G@ZIELT. ${ }^{1}$

\section{Big Data \& Co.}

Der Fortschritt von Wissenschaft und Technik erzeugt immer größere Datenmengen. So entschlüsseln heute Labore in kurzer Zeit vollständige DNA-Sequenzen durch neue Techniken wie „Whole Genome Sequencing“ oder „New Generation Sequencing“ (WGS/NGS). Diese Techniken nutzt man auch für die Aufklärung lebensmittelinduzierter Krankheitsausbrüche, indem man Bakterienisolate menschlicher Herkunft mit den entsprechenden Isolaten verdächtiger Lebensmittelproben vergleicht. Untersuchungsdaten könnten künftig in eine europäische Datenbank eingespeist werden, die eine Identifizierung mit dem Abgleich von Referenzmustern ermöglicht. So wären z.B. Lebensmittelfälschungen schneller identifiziert und die grenzüberschreitende Verfolgung von Lebensmittelbetrügern beschleunigt.

Big Data, künstliche Intelligenz und „Deep Learning“ sind weitere Entwicklungen, die auch am gesundheitlichen Verbraucherschutz nicht vorbeigehen. Sowohl die technische Ausstattung, wie auch die personellen Ressourcen müssen sich dem steigenden Umfang der Daten wie auch ihrer Komplexität anpassen und die Anforderungen an die Datensicherheit berücksichtigen. In der Arbeitswelt 4.0 entstehen neue Berufe, die zwischen den Fachprozessen und den technischen Anforderungen der Digitalisierung eine Brücke bilden.

Die Herausforderung besteht darin, steuerungsfähig zu bleiben. Dies kann nur gelingen, wenn flexible Strukturen entwickelt und erhalten werden. Zentrale Lösungen mit einer überschaubaren Anzahl von Standardprozessen und Rechenzentren sind politisch gewollt, stehen aber den Anforderungen von Fachprozessen häufig entgegen. Durch die sogenannte Blockchain-Technologie könnte man beispielsweise gezielt vernetzte Strukturen und Verschlüsselungstechnologien nutzen, um Informationen fälschungssicher über mehrere Stufen zu adressieren, etwa bei der Rückverfolgung von Warenströmen im Krisenfall.

Standardisierte Verwaltungsprozesse wie auch Behördenkommunikation lassen sich hingegen sinnvoll vereinheitlichen. Eine konsolidierte IT-Landschaft der Behörden einschließlich der Einführung der elektronischen Akte erlaubt Schnittstellen zum Verbraucher, die alle Eingaben und Anträge elektronisch stellen und direkt in

\footnotetext{
1 https://www.bvl.bund.de/DE/01_Lebensmittel/04_AntragstellerUn ternehmen/11_UeberwachungInternethandel/1m_ueberwachung_inter nethandel_node.html;jsessionid=CDC71AA94ABAEE5113B8924B6 8316DE2.1_cid332\#doc2633998bodyText1 Zugriff am 16. Oktober 2018.
}

den Bearbeitungsprozess einspeisen. Das BVL wird sich hier im Spannungsfeld von wissenschaftlicher Ausrichtung und behördlicher Erfordernisse positionieren müssen. Dabei sollen sowohl die sich aus der IT-Konsolidierung des Bundes ergebenden Chancen gestaltend genutzt, als auch Fach-IT-Strukturen aufgebaut werden.

In diesem Zusammenhang spielt die Frage nach der Möglichkeit von Open Data eine große Rolle. Die freie Nutzbarkeit von wissenschaftlichen Daten könnte zu mehr Transparenz führen. Die Datenbank des BVL verfügt über 120 Mio. Untersuchungsdaten aus der amtlichen Kontrolle der Bundesländer. Ein Teil davon wird jährlich im Rahmen von Berichtspflichten an die Europäische Lebensmittelsicherheitsbehörde (EFSA) übermittelt. Ab 2019 will die EFSA die von den Mitgliedstaaten übermittelten Lebensmittel-Überwachungsdaten als Forschungsdaten proaktiv veröffentlichen. Dieser gedankliche Ansatz könnte auch auf andere Bereiche übertragen werden: So könnte beispielsweise eine proaktive Veröffentlichung von Daten und Studien durch die Antragsteller bei der Zulassung eines Pflanzenschutzmittels die Transparenz in diesem sensiblen Bereich erhöhen. Im Gegenzug müssten diese Daten durch entsprechende gesetzliche Regelungen (vergleichbar mit dem Marken- und Patentschutz) vor einer unzulässigen Nutzung geschützt werden. Insgesamt sollte die vernetzende Rolle von Behörden als Knotenpunkt für den Austausch von Fachinformationen und Daten auf EU- und nationaler Ebene gestärkt werden und so zur Versachlichung politischer Konflikte beitragen.

Wie gestaltet sich der Ausblick? Die technologische Entwicklung wird voranschreiten, Quantencomputer werden mit ihrer enormen Leistungsfähigkeit in wenigen Jahren eine zentrale Rolle spielen. Gleichzeitig werden Komplexität und Umfang der Datenmengen weiter zunehmen. Die künstliche Intelligenz als Treiber von Digitalisierung und autonomer Systeme in allen Lebensbereichen wird eine Open-Data-Strategie zum festen Bestandteil von Behörden- und Firmenkulturen machen. Der Schutz des Verbrauchers durch Transparenz, Bereitstellung und Austausch von Informationen ist dabei das oberste Gebot.

\section{Literatur}

Schreiber GA (2013) Lebensmittelkontrolle und Verbraucherinformation im digitalen Zeitalter. J Verbr Lebensm 8:267-269 